\title{
Robust chimera states in SQUID metamaterials with local interactions
}

\author{
J. Hizanidis, N. Lazarides, and G. P. Tsironis \\ Crete Center for Quantum Complexity and Nanotechnology, Department of Physics, \\ University of Crete, P. O. Box 2208, 71003 Heraklion, Greece; \\ Institute of Electronic Structure and Laser, Foundation for Research \\ and Technology-Hellas, P.O. Box 1527, 71110 Heraklion, Greece; \\ National University of Science and Technology MISiS, Leninsky prosp. 4, Moscow, 119049, Russia
}

(Dated: September 28, 2018)

\begin{abstract}
We report on the emergence of robust multi-clustered chimera states in a dissipative-driven system of symmetrically and locally coupled identical SQUID oscillators. The "snake-like" resonance curve of the single SQUID (Superconducting QUantum Interference Device) is the key to the formation of the chimera states and is responsible for the extreme multistability exhibited by the coupled system that leads to attractor crowding at the geometrical resonance (inductive-capacitive) frequency. Until now, chimera states were mostly believed to exist for nonlocal coupling. Our findings provide theoretical evidence that nearest neighbor interactions are indeed capable of supporting such states in a wide parameter range. SQUID metamaterials are the subject of intense experimental investigations and we are highly confident that the complex dynamics demonstrated in this manuscript can be confirmed in the laboratory.
\end{abstract}

PACS numbers: 05.65.+b,05.45.Xt,78.67.Pt,89.75.-k,89.75.Kd

Keywords: local coupling, chimera state, attractor crowding

Since the first report on chimera states [1], the number of works dedicated to this phenomenon of coexisting synchronous and desynchronous oscillatory behavior has grown immensely (see [2] and references within). The counterintuitive nature of chimeras inspired Abrams and Strogatz [3] to name them after the mythological hybrid

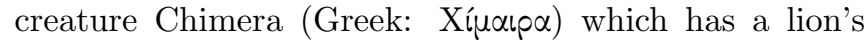
head, a goat's body and a snake's tail. The latest studies on chimera states focus on their stabilization and manipulation through various control techniques 4-7] and their experimental verification 8 -15].

Chimera states have mostly been found for nonlocal coupling between the oscillators 16 18. This fact has given rise to a general notion that nonlocal coupling is an essential ingredient for their existence. However, recently, it has been demonstrated that chimeras can be achieved for global coupling too [13, 19, 21]. The case of local coupling (i.e. nearest-neighbor interactions) has been studied less: In 22 chimera states were found in locally coupled networks, but the oscillators in the investigated systems were not completely identical. Very recently, the emergence of single- and double-headed (i. e. with one and two (in)coherent regions, respectively) chimera states in neural oscillator networks with local coupling has been reported 23. That system, however, is known to exhibit high metastability, which renders the chimera state non-stationary when tracked in long time intervals [24]. Here we demonstrate numerically the emergence of multi-clustered robust chimera states in SQUID metamaterials described in the local coupling approximation, in a relevant parameter region which has been determined experimentally in [25, 26.

Superconducting metamaterials comprising SQUIDs have been realized in both one and two dimensions [2530] and possess extraordinary properties such as negative magnetic permeability, dynamic multistability, broad- (a)

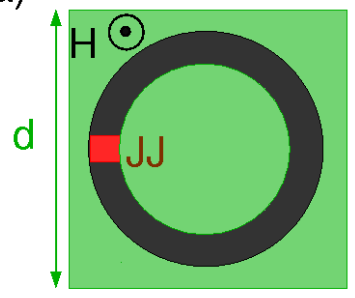

(b)

(c)

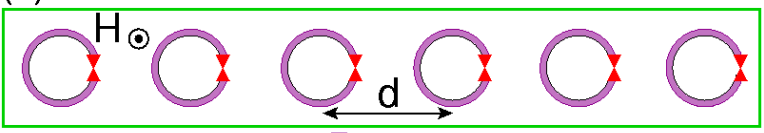

X Josephson junction Superconducting film

Figure 1. (Color online) Schematic of a SQUID in a magnetic field $\mathbf{H}(t)$ (a), and equivalent electrical circuit (b). The real Josephson junction is represented by the circuit elements in the brown-dashed box. (c) Schematic of a one-dimensional SQUID metamaterial.

band tunability, switching between different magnetic permeability states, as well as a unique form of transparency whose development can be manipulated through multiple parametric dependences. Some of these observed properties had been theoretically predicted both for the quantum [31] and the classical regime [32, 33. SQUID metamaterials are richly nonlinear effective media modeled by discrete phenomenological equations of coupled individual SQUID oscillators, which introduce qualitatively new macroscopic quantum effects into both the metamaterials and the coupled oscillator networks communities, i.e., magnetic flux quantization and the Josephson effect 34 .

A SQUID consists of a superconducting ring inter- 
rupted by a Josephson junction (JJ) as shown schematically along with its electrical equivalent circuit in Figs. 1(a) and 1(b), respectively; it is a highly nonlinear oscillator with a resonant response to an applied alternating (ac) magnetic field. When a periodic arrangement of $N$ identical SQUIDs is driven by a spatially uniform, ac field [Fig. 11(c)], its elements are coupled together through magnetic dipole-dipole forces which decay as the inverse cube of the distance. In the following, it is considered that each SQUID in the array is coupled only to its nearest neighbors, neglecting further-neighbor interactions. Then, the magnetic flux $\Phi_{n}$ threading the loop of the $n$th SQUID is

$$
\Phi_{n}=\Phi_{e x t}+L I_{n}+M\left(I_{n-1}+I_{n+1}\right),
$$

where $\Phi_{\text {ext }}$ is the external flux to each SQUID, $L$ is the self-inductance of the individual SQUID, $M$ is the mutual inductance between neighboring SQUIDs, and

$$
I_{n}=-C \frac{d^{2} \Phi_{n}}{d t^{2}}-\frac{1}{R} \frac{d \Phi_{n}}{d t}-I_{c} \sin \left(2 \pi \frac{\Phi_{n}}{\Phi_{0}}\right)
$$

is the current in the $n$th SQUID as provided by the resistively and capacitively shunted junction (RCSJ) model of the JJ 35, and $\Phi_{0}$ is the flux quantum. Within the RCSJ framework, $R, C$, and $I_{c}$ are the resistance, capacitance, and critical current of the JJ, respectively. Combining Eqs. (1) and (2), while neglecting all terms proportional to higher than the first power of the dimensionless coupling coefficient $\lambda=M / L[33$, gives

$$
\begin{array}{r}
\ddot{\phi}_{n}+\gamma \dot{\phi}_{n}+\phi_{n}+\beta \sin \left(2 \pi \phi_{n}\right)=\lambda\left(\phi_{n-1}+\phi_{n+1}\right) \\
+(1-2 \lambda) \phi_{a c} \cos (\Omega \tau)
\end{array}
$$

in which a sinusoidal external flux is considered. The flux through the $n$th SQUID loop $\phi_{n}$ and the amplitude of the external flux $\phi_{a c}$ are normalized to $\Phi_{0}$, the driving frequency $\Omega$ and the temporal variable $\tau$ (the overdots denote derivation with respect to $\tau$ ) are normalized to the geometrical (inductive-capacitive) resonance frequency of the SQUID $\omega_{L C}=1 / \sqrt{L C}$ and its inverse $\omega_{L C}^{-1}$, and $\beta=$ $\frac{I_{c} L}{\Phi_{0}}=\frac{\beta_{L}}{2 \pi}, \gamma=\frac{1}{R} \sqrt{\frac{L}{C}}$ is the SQUID parameter and loss coefficient, respectively.

The corresponding equation for a single SQUID is obtained from Eq. (3) by setting $\lambda=0$ and $\phi_{n}=\phi$. Then, by linearization of that equation and by neglecting dissipation and forcing, the SQUID resonance frequency can be obtained as $\Omega_{S Q}=\sqrt{1+\beta_{L}}$ in units of $\omega_{L C}$. The single SQUID equation for a certain range of parameters exhibits a "snake-like" resonance curve in which multiple stable and unstable periodic orbits coexist and vanish through saddle-node bifurcations of limit cycles. This dynamical behavior bears a big resemblance to the snaking bifurcation curves of localized structures reported in the Swift-Hohenberg equation [36].

The parameters which are responsible for the SQUID multistability are the loss coefficient $\gamma$ and the external ac flux $\phi_{a c}$. As $\gamma$ decreases and $\phi_{a c}$ increases, the snaking
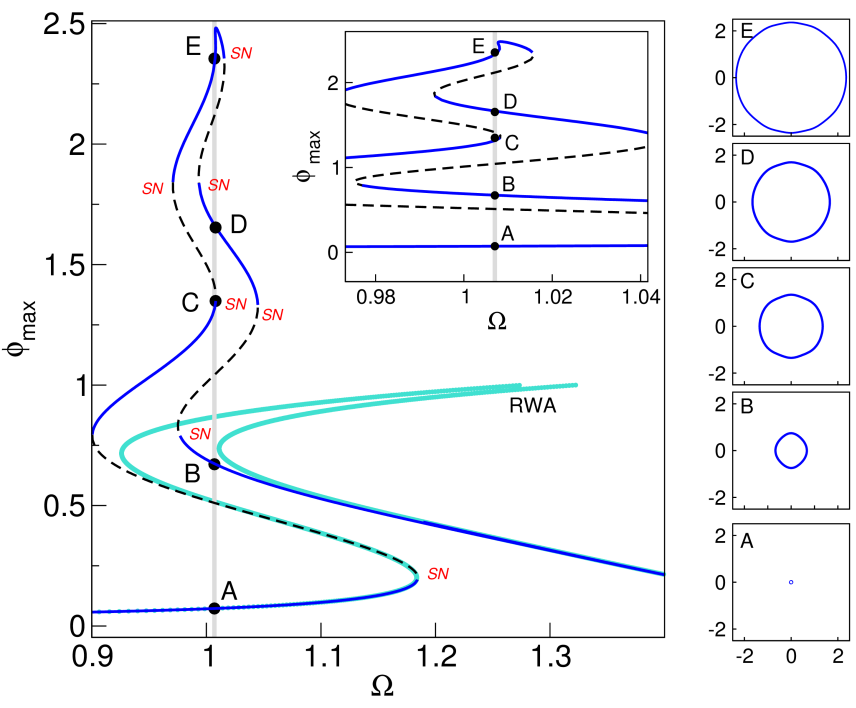

Figure 2. (Color online) The "snake-like" resonance curve of a single SQUID for $T=6.24(\Omega \simeq 1.007), \beta_{L}=0.86, \gamma=0.024$, and external ac flux $\phi_{a c}=0.06$. Solid blue and dashed lines correspond to branches of stable and unstable periodic solutions, respectively. Saddle-node bifurcations points are denoted as "SN". Thick gray line corresponds to $\Omega=1.007$ and turquoise lines are obtained from Eq. 4. Inset: Enlargement around the maximum multistability frequency. Phase portraits on the right show the corresponding periodic orbits of points $A-D$ on the resonance curve.

curve becomes more winding, achieving higher flux values and adding, thus, to the multistability. A typical such curve is shown in the left panel of Fig. 2 in which the amplitude of the flux variable $\phi_{\max }$ is plotted against the driving frequency $\Omega$. The blue solid lines correspond to the branches of stable periodic solutions while the dashed lines mark the unstable orbits. At each turning point where stable and unstable branches merge, a saddle node ("SN") bifurcation of limit cycles takes place. The inset figure shows a blow-up around $\Omega=1$ where the multistability is more prominent. This is illustrated by the intersections of the gray line with the snaking curve marked by the letters $A-D$. For this value of the driving frequency, we can distinguish $K=5$ coexisting periodic states of increasing amplitude; the corresponding orbits are shown in the phase portraits on the right.

An approximate resonance curve can be actually obtained from the single-SQUID equation using a truncated series expansion for $\sin (2 \pi \phi)$ with a trial solution $\phi=\phi_{m}(\tau) \cos [\Omega \tau+\theta(\tau)]$, where $\phi_{m}(\tau)$ and $\theta(\tau)$ are the slowly varying amplitude and phase, respectively. Then, by applying the rotating wave approximation (RWA) in which only the terms at the fundamental frequency $\Omega$ are retained, neglecting terms $\propto \ddot{\phi_{m}}, \ddot{\theta}, \dot{\theta}^{2}, \dot{\phi_{m}}, \dot{\theta}$, etc., and seeking for steady state solutions of the resulting alge- 
(a)

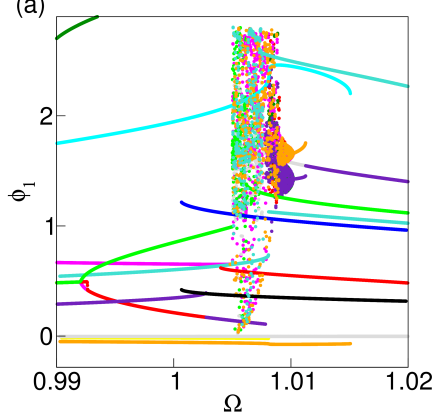

(b)

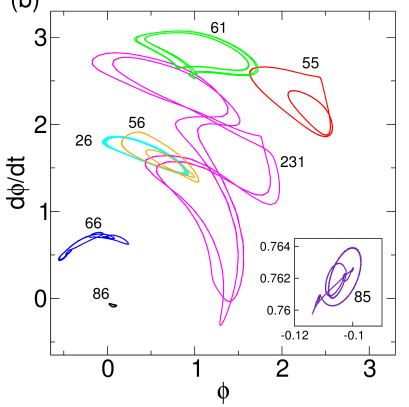

Figure 3. (Color online) (a) Solution branches for two coupled SQUIDs for an $\Omega$ scan around the maximum multistability point. At least ten (10) SQUID states are visible at this frequency. (b) $N=256$ coupled SQUIDs: Stroboscopic maps of some individual oscillators. Numbers denote the index of the respective SQUID oscillator. Coupling strength $\lambda=-0.025$ in both figures. All other parameters as in Fig. 2 .

braic system for $\phi_{m}(\tau)$ and $\theta(\tau)$, we get

$\Omega^{2}=\Omega_{S Q}^{2}-\beta_{L} \phi_{m}^{2}\left\{a_{1}-\phi_{m}^{2}\left[a_{2}-\phi_{m}^{2}\left(a_{3}-a_{4} \phi_{m}^{2}\right)\right]\right\} \pm \frac{\phi_{a c}}{\phi_{m}}$,

where $a_{1}=\pi^{2} / 2, a_{2}=\pi^{4} / 12, a_{3}=\pi^{6} / 144, a_{4}=$ $\pi^{8} / 2880$, which implicitly provides the sought $\phi_{m}(\Omega)$ relation, in which the first four terms in the series expansion are kept. The curves obtained from the earlier equation are shown in Fig. 2 in turquoise color, and reproduce the resonance curve up to $\phi_{m} \sim 0.6$ that includes the first saddle-node bifurcation.

In a metamaterial of $N$ weakly coupled SQUIDs there is a multiplicity of possible collective states that the system can reach, the number of which is of the order of $K^{N}$ or higher. The complexity in analyzing the behavior of such a system becomes clear by coupling together just two SQUIDs; the corresponding resonance curve maintains its "snake-like" form but with a thicker contour due to the additional (un)stable branches that are created (not shown here). Apart from the new periodic solutions, a number of coexisting chaotic attractors are also to be found. Figure 3 (a) shows a scan in $\Omega$ for values around the maximum multistability point, for two coupled SQUIDs. The different colors correspond to solution branches for different initial conditions and it is clear that around $\Omega=1.007$ the magnetic flux exhibits chaotic behavior. For $N=256$ coupled SQUIDs the complexity of the dynamics is even higher: Figure 3 (b) shows the stroboscopic maps corresponding to individual oscillators of a single configuration of the full system. The numbers next to the orbits denote the oscillator indices. This huge multiplicity of attractors is known as attractor crowding and has been observed before in coupled nonlinear oscillator systems [37.

Equations (3) are integrated numerically in time with a fourth-order Runge-Kutta algorithm with constant timestep and periodic boundary conditions, i. e., $\phi_{n}(\tau)=$ $\phi_{N+n}(\tau)$ for all $n$. The parameters used in the simu-

lations are close to the design parameters of the SQUID meta-atoms that make two-dimensional SQUID metamaterials [26, i.e., $L=60 \mathrm{pH}, C=0.42 \mathrm{pF}, I_{c}=4.7 \mu \mathrm{A}$, and subgap resistance $R=500 \Omega$, which give $\beta_{L}=0.86$ and $\gamma=0.024$ according to their definitions, while the value of the coupling coefficient between neighboring SQUIDs is $\lambda=-0.025$. The amplitude of the ac field is selected to be $\phi_{a c}=0.06$, within the experimentally accessible range $0.001-0.1$ 25. The selected values of $\gamma$ and $\phi_{a c}$ bring the SQUID metamaterial in the strongly nonlinear regime.

Figure 4 shows time-snapshots of the magnetic fluxes $\phi_{n}$ for different initial conditions and for two different values of the loss coefficient $\gamma$. The left panel is for $\gamma=0.024$ which is the value corresponding to the resonance curve of Fig. 2 The initial "sine wave" magnetic flux distribution for each simulation is shown by the gray solid line. The SQUIDs that are prepared at lower values form the coherent clusters of the chimera state, while those that are initially set at higher magnetic flux values oscillate incoherently. Moreover, as the "wave-length" of the initial magnetic flux distribution increases, so does the chimera state multiplicity (number of (in)coherent regions highlighted by the colored areas). Similar behavior is observed for lower values of the loss coefficient $(\gamma=0.0024)$ as shown in the right panel of Fig 4 . Here, the incoherent clusters are better illustrated since they are approximately of equal size and do not contain oscillators that "escape" from the incoherent cluster abiding around low magnetic flux values, something which is visible in the left panel. Furthermore, the coherent clusters (emphasized by the blue solid lines) are fixed around $\phi=0$, unlike in the left panel where additional clusters located at slightly higher values also form. Here we must recall that for low values of $\gamma$ (right panel) the winding of the "snake-like" resonance curve increases significantly creating, thus, new branches of stable (and equally unstable) periodic solutions. These branches are larger in number and smaller in size compared to those of higher $\gamma$ values (left panel). The lower amplitude branches which are the longer ones attract the SQUIDs that eventually form the coherent clusters. The other oscillators have a plethora of higher states to choose from and, therefore, create a more chaotic incoherent cluster than in the case of higher $\gamma$ values.

The observed chimera states can be quantified through the Kuramoto local order parameter [40] which is a measure for local synchronization:

$$
Z_{n}=\left|\frac{1}{2 \delta} \sum_{|j-n| \leq \delta} e^{i \phi_{j}}\right|, \quad n=1, \ldots, N .
$$

We use a spatial average with a window size of $\delta=5$ elements. A $Z_{n}$ value close to unity indicates that the $n$th SQUID belongs to the coherent cluster of the chimera state, while $Z_{n}$ is closer to 0 in the incoherent parts. In the left panel of Fig. 5 the space-time plots of the local order parameter corresponding to the chimera states 

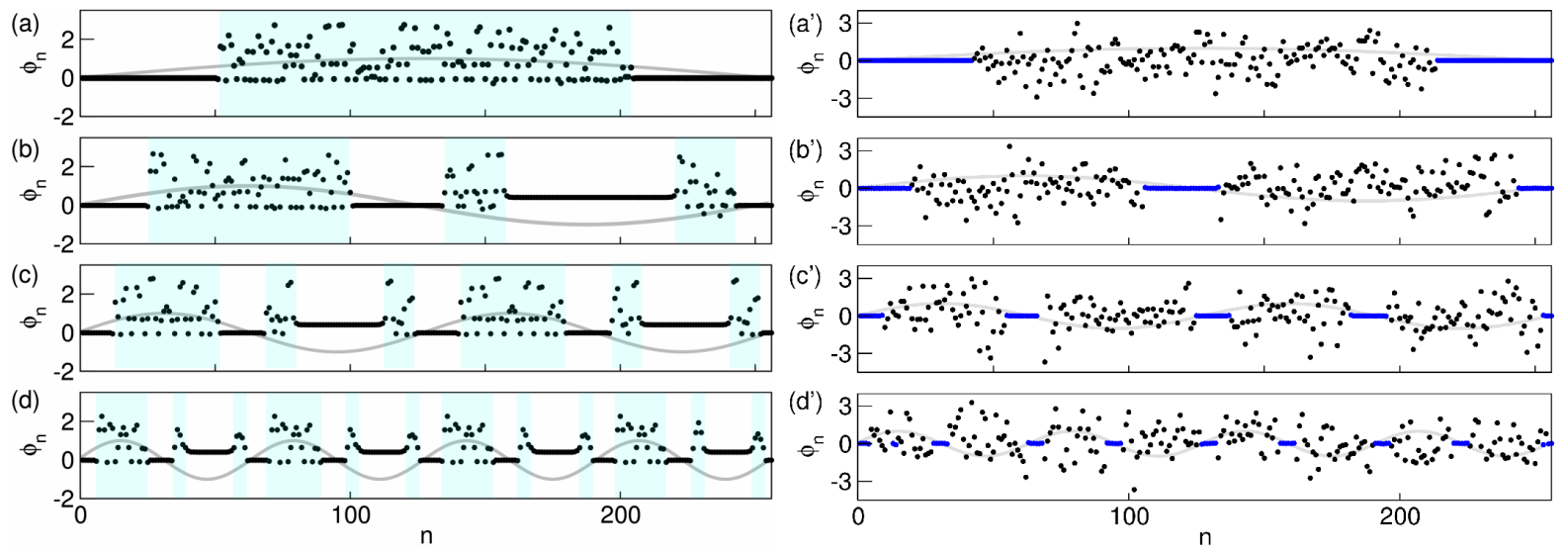

Figure 4. (Color online) Snapshots of the magnetic fluxes $\phi_{n}$ at time $T=5000$ for two different values of the loss coefficient: $\gamma=0.024$ in (a)-(d) and $\gamma=0.0024$ in (a')-(d'). Grey solid lines mark the initial magnetic flux distribution used in the simulations. Colored areas in the left panel mark the incoherent clusters while blue solid lines in the right panel emphasize the coherent clusters of the chimera states. All other parameters as in Fig. 2. See Supplemental Material for animations related to Figs. 4(b) 38 and (b') 39.
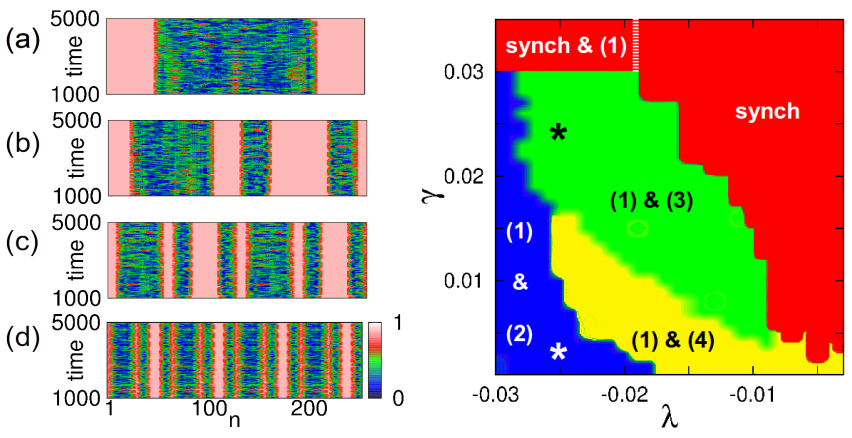

Figure 5. (Color online) Left panel: Space-time plots for the magnitude of the local order parameter $\left|Z_{n}\right|$ of the chimera states corresponding to Figs. 4(a)-(d). Right panel: Map of dynamic regimes in the $(\gamma, \lambda)$ parameter space for the initial conditions of Figs. 4(a) and (b). Numbers in brackets denote the multiplicity of the chimera state while "synch" stands for (route to) synchronization. All other parameters as in Fig. 2

of Fig. 4(a)-(d) are shown. The number of (in)coherent regions increases according to the initial conditions and the size and location of the clusters is constant in time. Previous works on SQUID metamaterials showed that for nonlocal coupling single and double-headed chimera states coexist with solitary states [41] and metastable states of drifting (in)coherence [40, 42]. Note that in these studies the focus was on a different dynamical area with the external driving frequency lying outside the multistability regime. For a suitable choice of $\Omega$, stable chimera states can be achieved for nonlocal coupling also. However, they exist only for low coupling strengths $\lambda$; the threshold value of the coupling strength in the case of local coupling is much higher. Local coupling is therefore crucial for the emergence of robust chimera states, both in structure and in lifetime, for a wide range of parameters.

Previously we stressed the importance of multistability and the impact of the loss coefficient $\gamma$ in the formation of chimeras in our system. In addition to that, it is important to note the role of the network topology which is defined through the local nature of interactions and the coupling strength $\lambda$. As already shown in Fig. 4. our system exhibits a variety of coexisting multi-clustered chimera states. A systematic study in the $(\lambda, \gamma)$ parameter space is depicted in the right panel of Fig. 5 , where the observed patterns for two different sets of initial conditions (namely those of Fig. $4\left(\mathrm{a}, \mathrm{a}^{\prime}\right)$ and (b,b')) are mapped out. The numbers in the brackets correspond to the multiplicity of the respective chimeras and "synch" denotes the synchronized states. The black and white asterisk mark the $(\lambda, \gamma)$ values used in the left and right panel of Fig. 4. respectively. We see that for low values of the loss coefficient and for low and medium (in absolute value) coupling strengths, single- and four-headed chimera states coexist (yellow area). As $\gamma$ increases, the effect of multistability diminishes and the system enters the synchronized state (red area) either directly or through a region where three-headed chimeras coexist with single-headed ones (green area). For stronger couplings (blue area), double-headed chimeras coexist with single chimeras. The latter persist also for high $\gamma$ values where the synchronized state is achieved. For initial conditions with a larger modification in space (like in Fig. 4(c,c') and (d,d')) chimera states with higher multiplicity can be found, but the mechanism towards synchronization is the same: the fully coherent state is reached through the appearance of solitary states [40, 41].

In conclusion, the model equations for a SQUID metamaterial truncated to only nearest-neighbor coupling were integrated in time with properly chosen initial con- 
ditions that allow the system to reach chimera states. These novel states emerge due to the extreme multistability that leads to attractor crowding at the geometrical resonance frequency. Typical chimera states are presented and characterized with respect to their local synchronization level. A systematic study in the relevant parameter space reveals the coexistence of multiheaded chimeras and the oscillators for a metamaterial in a chimera state elucidate a number of different trajectories, some of which are chaotic. Since chimera states have been intimately connected with nonlocal coupling, the present results point towards the need to revise the general consensus on the essential conditions for their ex- istence.

\section{Acknowledgement}

This work was partially supported by the European Union Seventh Framework Programme (FP7-REGPOT2012-2013-1) under grant agreement $\mathrm{n}^{\circ} 316165$, and the Ministry of Education and Science of the Russian Federation in the framework of the Increase Competitiveness Program of NUST "MISiS" (No. K2-2015-007). J. H. would like to thank Thomas Isele and Yuri Maistrenko for valuable discussions.
[1] Y. Kuramoto and D. Battogtokh, Nonlinear Phenom. Complex Syst. 5, 380 (2002).

[2] M. J. Pannagio and D. Abrams, Nonlinearity 28, R67 (2015).

[3] D. M. Abrams and S. H. Strogatz, Phys. Rev. Lett. 93, $174102(2004)$.

[4] J. Sieber, O. E. Omel'chenko, and M. Wolfrum, Phys. Rev. Lett. 112, 054102 (2014).

[5] C. Bick and E. A. Martens, New J. Phys. 17, 033030 (2015).

[6] I. Omelchenko, O. E. Omel'chenko, A. Zakharova, M. Wolfrum, and E. Schöll, Phys. Rev. Lett. 116, 114101 (2016).

[7] T. Isele, J. Hizanidis, A. Provata, and P. Hövel, Phys. Rev. E 93, 022217 (2016).

[8] M. R. Tinsley, S. Nkomo, and K. Showalter, Nature Phys. 8, 662 (2012).

[9] A. M.Hagerstrom, T. E. Murphy, R. Roy, P. Hövel, I. Omelchenko, and E. Schöll, Nature Phys. 8, 658 (2012).

[10] M. Wickramasinghe and I. Z. Kiss, PLoS ONE 8, e80586 (2013).

[11] E. A. Martens, S. Thutupalli, A. Fourrière, and O. Hallatschek, Proc. Nat. Acad. Sciences 110, 10563 (2013).

[12] D. P. Rosin, D. Rontani, N. D. Haynes, E. Schöll, and D. J. Gauthier, Phys. Rev. E 90, 030902 (2014).

[13] L. Schmidt, K. Schönleber, K. Krischer, and V. Garc 'aMorales, Chaos 24, 013102 (2014).

[14] L. V. Gambuzza, A. Buscarino, S. Chessari, L. Fortuna, R. Meucci, and M. Frasca, Phys. Rev. E 90, 032905 (2014).

[15] T. Kapitaniak, P. Kuzma, J. Wojewoda, K. Czolczynski, and Y. Maistrenko, Sci. Rep. 4, 6379 (2014).

[16] I. Omelchenko, O. E. Omel'chenko, P. Hövel, and E. Schöll, Phys. Rev. Lett. 110, 224101 (2013).

[17] A. Zakharova, M. Kapeller, and E. Schöll, Phys. Rev. Lett. 112, 154101 (2014).

[18] I. Omelchenko, A. Provata, J. Hizanidis, E. Schöll, and P. Hövel, Phys. Rev. E 91, 022917 (2015).

[19] G. C. Sethia and A. Sen, Phys. Rev. Lett. 112, 144101 (2014).

[20] A. Yeldesbay, A. Pikovsky, and M. Rosenblum, Phys. Rev. Lett. 112, 144103 (2014).

[21] F. Böhm, A. Zakharova, E. Schöll, and K. Lüdge, Phys. Rev. E 91, 040901(R) (2015).
[22] C. Laing, Phys. Rev. E 92, 050904(R) (2015).

[23] B. K. Bera, D. Ghosh, and M. Lakshmanan Phys. Rev. E 93, 012205 (2016).

[24] J. Hizanidis, N. E. Kouvaris, G. Zamora-López, A. DíazGuilera, and C. G. Antonopoulos, Sci. Rep. 6, 19845 (2016).

[25] M. Trepanier, D. Zhang, O. Mukhanov, and S. M. Anlage, Phys. Rev. X 3, 041029 (2013).

[26] D. Zhang, M. Trepanier, O. Mukhanov, and S. M. Anlage, Phys. Rev. X 5, 041045 (2015).

[27] S. Butz, P. Jung, L. V. Filippenko, V. P. Koshelets, and A. V. Ustinov, Opt. Express 21, 22540 (2013).

[28] P. Jung, S. Butz, M. Marthaler, M. V. Fistul, J. Leppäkangas, V. P. Koshelets, and A. V. Ustinov, Nat. Commun. 5, 3730 (2014).

[29] P. Jung, A. V. Ustinov, and S. M. Anlage, Supercond. Sci. Technol. 27, 073001 (2014).

[30] A. V. Ustinov, IEEE Trans. Terahertz Sci. Technol. 5, 22 (2015).

[31] C. Du, H. Chen, and S. Li, Phys. Rev. B 74, 113105 (2006).

[32] N. Lazarides and G. P. Tsironis, Appl. Phys. Lett. 90, 163501 (2007).

[33] N. Lazarides and G. P. Tsironis, Supercond. Sci. Technol. 26, 084006 (2013).

[34] B. Josephson, Phys. Lett. A 1, 251 (1962).

[35] K. K. Likharev Dynamics of Josephson Junctions and Circuits, Gordon and Breach, Philadelphia (1986).

[36] G. Kozyreff and S. J. Chapman, Phys. Rev. Lett. 97, 044502 (2006).

[37] K. Wiesenfeld and P. Hadley, Phys. Rev. Lett. 62, 1335 (1989).

[38] Supplemental video SM1

[39] Supplemental video SM2

[40] J. Hizanidis, N. Lazarides, G. Neofotistos, and G. P. Tsironis, Eur. Phys. J. Special Topics, Springer 225 (5) (2016) (in print).

[41] P. Jaros, Y. Maistrenko, and T. Kapitaniak, Phys. Rev. E 91, 022907 (2015).

[42] N. Lazarides, G. Neofotistos, and G. P. Tsironis, Phys. Rev. B 91, 054303 (2015). 